\title{
Anti-Ma2 antibody related paraneoplastic limbic/brain stem encephalitis associated with breast cancer expressing Ma1, Ma2, and Ma3 mRNAs
}

\author{
K Sahashi, K Sakai, K Mano, G Hirose
}

J Neurol Neurosurg Psychiatry 2003;74:1332-1335

A 69 year old woman presented with cognitive impairment and supranuclear gaze palsy caused by paraneoplastic limbic/brain stem encephalitis associated with atypical medullary breast carcinoma. The cerebrospinal fluid from the patient harboured an anti-neuronal cell antibody against Ma2 antigen, but not against Mal or Ma3 antigen. Despite the antibody being restricted to the Ma2 antigen, the patient's cancer tissue expressed Mal, Ma2, and Ma3 mRNAs. These results, and the expression of Ma2 mRNA in an atypical medullar breast carcinoma in another patient without paraneoplastic encephalitis, indicate that the induction of anti-Ma2 antibody depends on host immunoreponsiveness and not on the presence of the antigen itself in the cancer.

$\mathrm{P}$ araneoplastic neurological syndromes are neurological degenerative disorders that occur in patients with neoplasms outside the nervous system, associated with anti-neuronal cell antibodies that provide antitumour immunity. Fifty per cent of the primary tumours associated with paraneoplastic limbic encephalitis are small cell lung carcinomas, and in about half of these there are antibodies against the Hu family of neuronal RNA binding proteins. ${ }^{1-3}$ Onconeuronal proteins Mal, Ma2, and Ma3 have been identified as antigens for paraneoplastic encephalitis. ${ }^{4-6}$ A review of 29 patients with anti-Ma (Mal, Ma2, and Ma3) antibodies showed that anti-Ma2 antibody was present in all the cases, and 18 patients carried anti-Ma2 antibody alone. ${ }^{6-8}$ In those 18 patients, a testicular tumour was present in 14 cases; other associated tumours included breast cancer, lung cancer, and follicular lymphoma.

Here we report the clinical and immunopathological findings in a female patient who presented with paraneoplastic limbic/brain stem encephalitis associated with atypical medullary breast carcinoma. The patient carried the antibody against Ma2 antigen, but not Mal or Ma3 antigen, and the cancer tissue expressed Mal, Ma2, and Ma3 mRNAs.

\section{CASE REPORT}

A 69 year old woman was referred to our hospital for progressive amnesia and hypersomnia lasting for five months. She had also had diplopia and visual hallucinations for three months. She had had a total hysterectomy for uterine fibroids when she was 50. On admission in January 2002, her axillary temperature was $38.0^{\circ} \mathrm{C}$ and she had moderate depression, agitation, visual hallucination, amnesia, and hypersomnia. She had lost orientation in place and time, and her short term memory was severely impaired. Epileptic seizures were not documented. The pupils were equal in size and the light reflexes were spared. Her left eye was exophoric at rest, and its adduction was moderately impaired. She had supranuclear gaze palsy and her vertical gaze was completely impaired bilaterally. There were no pyramidal tract signs, cerebellar ataxia, or bulbar dysfunction. Muscle strength and sensation were normal.

Routine laboratory tests showed no abnormalities. These included tumour markers of carcinoembryonic antigen (CEA), neurone specific enolase (NSE), and squamous cell carinoma related antigen (SCC) thyroid function, vitamin B-1, antinuclear antibodies, and rheumatoid factor. Both T2 weighted and FLAIR magnetic resonance imaging (MRI) showed areas of high signal strength in the bilateral mesial temporal lobes, the amygdala, the hippocampus, and the hypothalamus. There was no region of mass effect or gadolinium enhancement. The cerebral cortex and brain stem were not affected. ECD-SPECT showed prominent hypoperfusion in the frontal lobes, and somewhat less bilaterally in the medial temporal lobes. The cerebrospinal fluid (CSF) contained 4 lymphocytes $/ \mathrm{mm}^{3}$ and 20 $\mathrm{mg} / \mathrm{dl}$ protein, and the cytology was negative. IgM titres of CSF for herpes simplex were normal. Chest computed tomography (CT) revealed swelling of the right axillary lymph nodes but no lung cancer. Echo mammography showed a $30 \mathrm{~mm}$ hypoechogenic mass in the right breast, which was not palpable, and a needle biopsy of the lesion revealed adenocarcinomatous cells. A diagnosed of paraneoplastic limbic/brain stem encephalitis associated with breast cancer was made and the patient underwent total right mammectomy.

Even after resection of the cancer, however, amnesia, hypersomnia, and delirium showed further deterioration. Intravenous methylprednisolone $1 \mathrm{~g}$ /day for three days followed by oral prednisolone $1 \mathrm{mg} / \mathrm{kg} /$ day markedly improved her cognitive impairment, up to the level on admission. Her gaze palsies, however, remained unchanged. The patient was essentially stabilised, but was still suffering from amnesia, hypersomnia, hallucination, and gaze palsy without recurrence of the cancer. Repeat brain MRI showed no change in the signal abnormalities.

\section{LABORATORY ANALYSES}

\section{Recombinant Ma1, Ma2, and Ma3 proteins}

The Mal, Ma2, or Ma3 full length cDNA was subcloned into a pGEX 5X-3 vector (Amersham Bioscience, Piscataway, New Jersey, USA). The GST fused proteins were induced with isopropyl- $\beta$-D-thio-galactoside and purified by Sephadex 4B gel (Amersham Bioscience).

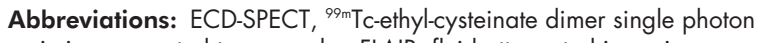
emission computed tomography; FLAIR, fluid attenuated inversion recovery; GST, glutathione-S-transferase; mRNA, messenger RNA; PCR, polymerase chain reaction; RT-PCR, reverse transcriptase polymerase chain reaction; SDS-PAGE, sodium dodecyl sulphate polyacrylamide gel electrophoresis 
Table 1 Polymerase chain reaction primers used for the first and nested RT-PCR

\begin{tabular}{lll}
\hline PC & Forward & Reverse \\
\hline Mal first & 5'-AAATGGCGATGACACTG-3' & 5'-GCTGCATTGCAGTCGTTCAC-3' \\
Mal nested & 5'-AGTCTCCCAAACGCCGCTC-3' & 5'-GCTGCATTGCAGTCGTTCAC-3' \\
Ma2 first & 5'-CCATGGCGCTGGCACTGTTAGA-3' & 5'-CGGCCATAGCCATCTCGTTCC-3' \\
Ma2 nested & 5'-CTGGACCTCACTGGGAATGG-3' & 5'-CGGCCATAGCCATCTCGTTCC-3' \\
Ma3 first & 5'-CCATGCCGTTGACCTTGTTA-3' & 5'-GCAAATGTTGCCCTGTTCTC-3' \\
Ma3 nested & 5'-CAGATACAGGGTGATTGGCA-3' & 5'-GCAAATGTTGCCCTGTTCTC-3' \\
\hline RT-PCR, reverse transcriptase polymerase chain reaction. & \\
\hline
\end{tabular}

\section{Immunohistochemical analysis}

Cryostat sections of rat cerebrum were incubated with horse serum and then with CSF from the patient diluted 1:10. The sections were reacted with biotinylated goat anti-human IgG and then with the Vectastain ABP complex (Vector Laboratories, Burlingame, California, USA), after which they were developed in deiamminobenzidine tetrahydrochloride.

\section{Western blot analysis}

Recombinant Mal, Ma2, and Ma3 proteins were separated by SDS-PAGE and electrophoretically transferred to nitrocellulose filters. The blots were reacted with CSF from the patient, then with alkaline phosphatase conjugated anti-human IgG antibody, and subsequently developed using nitroblue tetrazolium chloride and 5-bromo-4-chloro-3-indolylphosphate p-toluidine salt.

\section{Reverse transcriptase polymerase chain rection}

One microgram of total RNA was extracted from the deparaffinised cancer specimens using Trizol (Invitrogen, Carlsbad, California, USA), and reversibly transcribed with MMLV reverse transcriptase. The cDNAs were amplified by first and nested polymerase chain reaction (PCR) using primers specific to Mal, Ma2, or Ma3 mRNA (table 1).
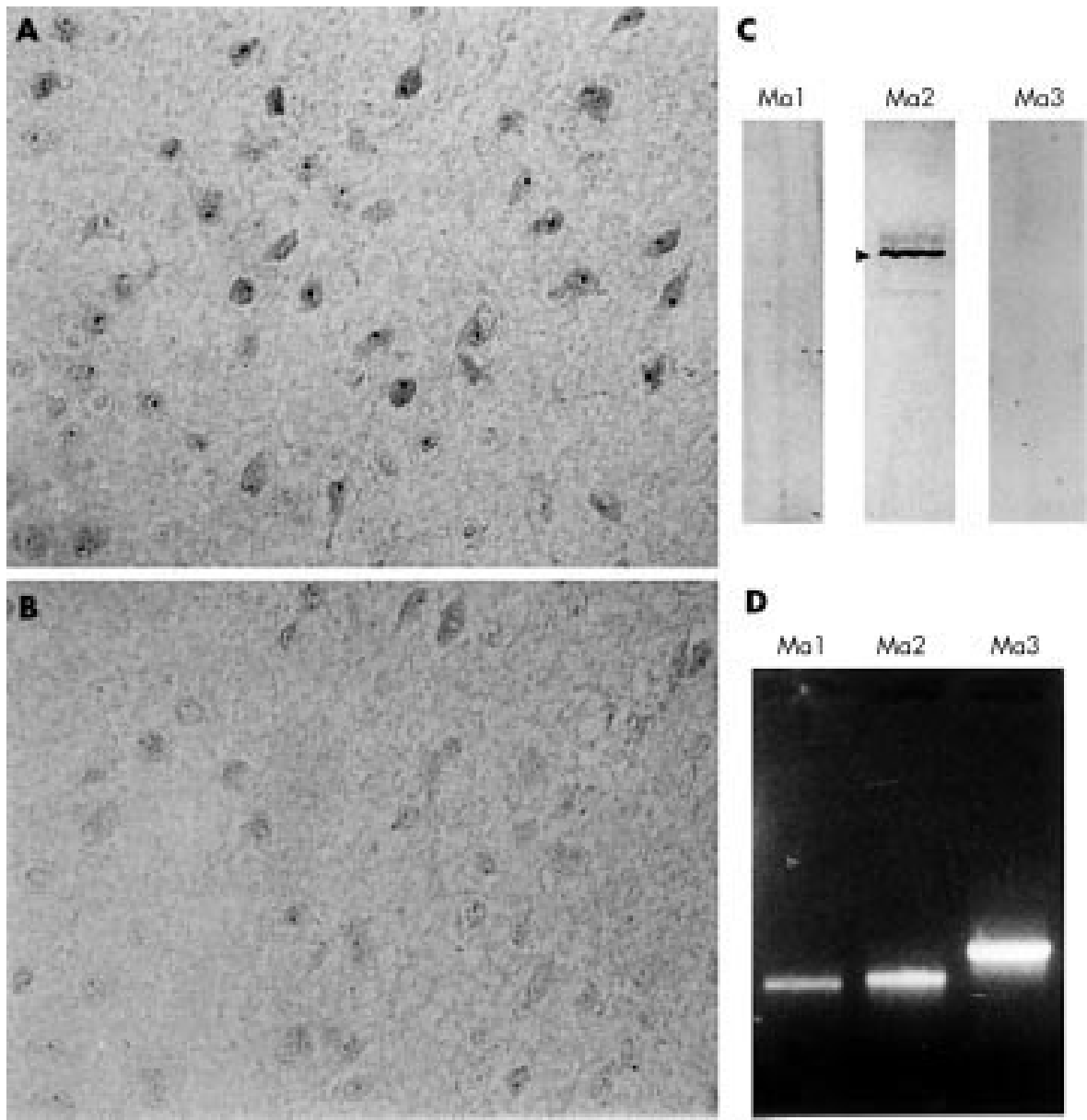

D

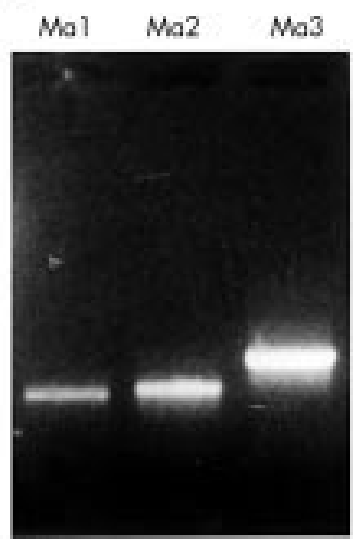

Figure 1 (A) Immunohistochemical analysis for the expression of Ma2 antigen (original magnification, $\times 400$ ). Frozen sections of rat cerebrum were reacted with cerebrospinal fluid (CSF; diluted to 1:10) from the patient. The reactivity occurred mainly in the nuclei, and was concentrated in the nucleoli and to a lesser extent in the cytoplasm. (B) The intensity of the antibody response in immunohistochemistry using CSF (diluted to 1:10) from the patient apparently decreased after corticosteroid treatment. (C) Western blot analysis of recombinant Mal Ma2, and Ma3 proteins with CSF (diluted to 1:1) from the patient. The CSF reacted with Ma2 protein, but not with Mal or Ma3 protein. (D) RT-PCR of RNA from the patient's cancer tissue using Mal, Ma2, or Ma3 specific primers. All Mal, Ma2, and Ma3 mRNAs were shown by electrophoresis to be transcribed on the tissue. RT-PCR, reverse transcriptase polymerase chain reaction. 


\section{RESULTS}

Immunohistochemistry of rodent brain tissue with CSF from the patient showed the presence of antineuronal cell antibodies that reacted predominantly with the nuclei in a dot-like pattern, and to a lesser extent with the cytoplasm of many neurones in the cerebrum, including the hippocampus (fig 1A). The CSF did not react with neurones in the cerebellum. These immunohistochemical findings are compatible with those of anti-Ma2 antibody. The intensity of the antibody responses in the patient's CSF decreased after corticosteroid treatment, as shown in fig $1 \mathrm{~B}$. Western blot analysis showed that patient's CSF reacted only with recombinant Ma2 protein, but not with recombinant Mal or Ma3 protein (fig lC). These results indicate that the antibody in the CSF from the patient is anti-Ma2 antibody.

Histological examination showed that the breast cancer was an atypical medullary carcinoma. In order to investigate the expression of Ma antigens in the cancer tissue, we extracted total RNA from the tissue and amplified it by RT-PCR using primers specific to Mal, Ma2, or Ma3 mRNA. The result indicated the expression of all the Ma transcripts in the cancer tissue (fig 1D). Ma2 mRNA has been detected in the tissue of atypical medullary breast carcinoma from another patient without neurological symptoms or anti-neuronal cell antibodies (data not shown). We deparaffinised the paraffin embedded sections of the patient's breast cancer tissue and investigated them for antigen expression by immunohistochemistry. However, the background reactivity was so high that we could not determine the expression of Ma2 antigen in the specimens.

\section{DISCUSSION}

We report a case of paraneoplastic limbic/brain stem encephalitis with anti-Ma2 antibody associated with breast cancer. In our patient, breast cancer was not detectable by detailed physical examination. Screening for lung cancer by chest CT showed swelling of the axillary lymph nodes and led to the diagnosis of breast cancer. Subsequently, we identified an anti-neuronal cell antibody in the patient's CSF, and made a diagnosis of paraneoplastic encephalitis.

Our patient shows the typical clinical and radiographic features of Ma2 related limbic/brain stem encephalitis. ${ }^{56}$ Her cognitive dysfunction improved markedly following the administration of corticosteroids, though anti-tumour treatment had not been effective in achieving this. A reduced antiMa2 antibody titre probably accounted for the clinical improvement. Although MRI revealed no brain stem lesions, the vertical supranuclear gaze palsy was probably caused by concurrent brain stem encephalitis and was refractory to the anti-tumour and immunomodulating treatments.

This is the second report of anti-Ma2 antibody related paraneoplastic limbic/brain stem encephalitis associated with atypical medullary breast carcinoma. Sutton et al reported the first case, in which there was similar cognitive dysfunction, ${ }^{7}$ though their patient did not have brain stem encephalitis. Their patient carried anti-Ma2 antibody and a marginal amount of anti-Mal antibody, whereas our patient harboured anti-Ma2 antibody exclusively. The similarity of these two patients implies that atypical medullary breast carcinoma readily triggers anti-Ma2 antibody production.

Mal, Ma2, and Ma3 proteins are homologous and probably play a role in mRNA biogenesis. ${ }^{6}$ Mal protein is expressed in brain and testis, Ma2 only in brain, and Ma3 in brain, testis, and faintly in some other organs, ${ }^{4-6}$ but the normal Ma proteins do not raise anti-Ma antibodies. Although anti-Ma2 antibody is a useful marker in identifying paraneoplastic encephalitis, it remains to be proven whether the antibody plays a crucial role in the pathogenesis, and the mechanism of production of the antibody is unknown.
We found lower titres of anti-Ma2 antibody in the patient's serum than in the CSF. In paraneoplastic neurological syndromes, especially those associated with encephalomyelitis, titres of anti-neuronal cell antibody are often higher in the CSF than in the serum, and intrathecal production of the antibodies has been suggested. ${ }^{9}$ This may be the case with the Ma2 antibody associated limbic/brain stem encephalitis in the present study.

Expression of onconeuronal protein could be under the control of post-transcriptional regulatory mechanisms, and a discrepancy sometimes occurs between mRNA and protein expression. ${ }^{10}{ }^{11}$ The possibility cannot be excluded that there is a discrepancy between Ma mRNAs and protein expression in our patient's cancer tissue. Although we could not demonstrate expression of the Ma2 protein in our patient's cancer tissue, we observed parallel expression of the Ma2 mRNA and protein in various cancer cell lines (unpublished observation), indicating that Ma2 protein unlikely undergoes the posttranscriptional regulation of expression. This also implies that expression of Ma2 is not restricted to this patient's cancer, but occurs in a broad range of cancers.

Ma2 mRNA was expressed in atypical medullary breast carcinoma in another patient without paraneoplastic encephalitis or anti-Ma antibodies. Taken together, these results indicate that the expression of the antigen is not sufficient to induce anti-Ma antibodies, and that the induction of anti-Ma2 antibody could depend on active host immunoresponsiveness. Further investigations will be needed to determine the sequences of the antigen in the cancer and the host immunoresponsiveness, including cellular immunity and HLA typing.

\section{ACKNOWLEDGEMENTS}

This work was supported in part by a grant for neuroimmunological disease research from the Ministry of Health and Welfare of Japan, and a grant for project research from the High Technology Centre of Kanazawa Medical University (H2003-3). We are grateful to Dr Kinji Ohno, Neurology Research, Mayo Clinic, Rochester, Minnesota, USA for critical input and discussions.

\section{Authors' affiliations}

K Sahashi, K Mano, Department of Neurology, Japanese Red Cross Nagoya First Hospital, Aichi, Japan

K Sakai, G Hirose, Department of Neurology, Kanazawa Medical University, Ishikawa, Japan

Correspondence to: Dr K Sahashi, Department of Neurology, Japanese Red Cross Nagoya First Hospital, 3-35 Michishita-cho, Nakamura-ku, Nagoya 453-851 1, Japan; sahashi@gc5.so-net.ne.jp

Received 21 January 2003

In revised form 19 February 2003

Accepted 23 February 2003

\section{REFERENCES}

1 Graus F, Keime-Guibert F, Reñe R, et al. Anti-Hu-associated paraneoplastic encephalomyelitis: analysis of 200 patients. Brain $2001 ; 124: 1138-48$

2 Szabo A, Dalmal J, Manley G, et al. HuD, a paraneoplastic encephalomyelitis antigen, contains RNA-binding domains and is homologous to Elav and sex-lethal. Cell 1991;67:325-33.

3 King PH, Dropcho EJ. Expression of Hel-N1 and Hel-N2 in small-cell lung carcinoma. Ann Neurol 1996;39:679-71

4 Voltz R, Gultekin SH, Rosenfeld MR, et al. A serologic marker of paraneoplastic limbic and brain-stem encephalitis in patients with testicular cancer. N Engl J Med 1999;340:1788-95.

5 Gultekin SH, Rosenfeld MR, Voltz R, et al. Paraneoplastic limbic encephalitis: neurological symptoms, immunological findings and tumour association in 50 patients. Brain 2000;123:1481-94.

6 Rosenfeld MR, Eichen JG, Wade DF, et al. Molecular and clinical diversity in paraneoplastic immunity to Ma proteins. Ann Neurol 2001;50:339-48

7 Sutton I, Winter J, Rowlands D, et al. Limbic encephalitis and antibodies to Ma2: a paraneoplastic presentation of breast cancer. J Neurol Neurosurg Psychiatry 2000;69:266-8. 
8 Barnett M. Prosser J, Sutton I, et al. Paraneoplastic brainstem encephalitis in a woman with anti-Ma2 antibody. J Neurol Neurosurg Psychiatry $2001 ; 70: 222-5$

9 Vega FG, Graus QM, Chen MP, et al. Intrathecal synthesis of the anti-Hu antibody in patients with paraneoplastic encephalomyelitis or sensory neuropathy: clinical-immunologic correlation. Neurology 1994:44:2145-7.
10 Sakai K, Kitagawa Y, Ogasawara $T$, et al. Expression of messenge RNA encoding a paraneoplastic cerebellar degeneration-associated antigen in the rat hippocampus. Neurosci Lett 1995;197:33-6.

11 Corradi JP, Yang C, Darnell, et al. A post-transcriptional regulatory mechanism restricts expression of the paraneoplastic cerebellar degeneration antigen $\mathrm{cdr} 2$ to immune privileged tissues. J Neurosci 1997; 17:1406-15.

\section{NEUROLOGICAL PICTURE}

\section{Cerebrotendinous xanthomatosis}

A

37 year old patient was admitted for a generalised seizure. He was treated with valproic acid for partial epilepsy. Neurological examination revealed a cerebellar and pyramidal syndrome, moderate mental retardation, and enlargement of Achilles tendons xanthomas (figs 1 and 2).

MRI showed capsular, putaminal, and symmetrical cerebellar hyper intense signal on T2 images and Tc labelled hexamethylpropyleneamine oxime (HMPAO) SPECT illustrated temporal hypoperfusion. EEG revealed diffuse slow waves with bursts of high voltage shapes waves. Electromyography was suggestive of axonal polyneuropathy. CFS was normal, including isoelectric focusing. Complete blood counts, serum cholesterol, urea, glucose, electrolytes, proteins, liver function tests, vitamin B12, and angiotensin converting enzyme were all normal. Serology for HIV, herpes simplex, Ebstein-Barr virus, cytomegalovirus, HTLV I-II and TPHA test and VDRL test were all negative. Cholestanol in serum was increased to $44.33 \mu \mathrm{mol} / \mathrm{l}$ (20 times normal value) evoking cerebrotendinous xanthomatosis (CTX). Pathogenic mutations $1183 \mathrm{C}>\mathrm{T}$ and 1209C $>$ G (N403K, exon 7, heterozygous) were detected in the CYP27Al analysis-mutations that has not been described before. Chenodeoxycholique acid was introduced.

The CTX is a rare recessive autosomal disease caused by mutations of the sterol 27 hydroxylase gene. ${ }^{1}$ CTX patients have elevated plasma levels of cholestanol byproduct of abnormal bile acid synthesis. Clinical hallmarks are tendon xanthomas, juvenile cataract, and nervous system abnormalities. The biochemical diagnosis is made by determination of the serum cholestanol level and by the determination of bile alcohol excretion in urine.

About 300 cerebrotendinous patients throughout the world have been reported, but it is our contention that there are many undiagnosed patients. A diagnosis is crucial because chenodeoxycholic acid treatment may stop the deterioration and improve the neurological functions. ${ }^{3}$ We report a patient presenting a mental retardation, cerebella ataxia, and epilepsy. Enlargement of Achilles tendons bilaterally observation leaded to CTX diagnosis.

\section{G Castelnovo, L Jomir, S Bouly Department of Neurology, Caremeau Hospital, CHU Nimes,} France

For correspondence: G Castelnova, Department of Neurology, Caremeau Hospital, CHU Nimes, France; giovanni.castelnova@chu_nimes.fr

\section{References}

1 Cali JJ, Hsieh CL, Francke U, et al. Mutations in the bile acid biosynthetic enzyme sterol 27-hydroxylase underlie cerebrotendinous xanthomatosis. J Biol Chem 1991;266: 7779-83.

2 Wolthers BG, Walrecht HT, van der Molen JC, et al. Use of determinations of 7-lathosterol (5 alpha-cholest-7-en-3 beta-ol) and other cholesterol precursors in serum in the study and treatment of disturbances of sterol metabolism, particularly cerebrotendinous xanthomatosis. J Lipid Res 1991;32:603-12.

3 Berginer VM, Salen G, Shefer S. Long-term treatment of cerebrotendinous xanthomatosis with chenodeoxycholic acid. N EnglJ Med 1984;311:1649-52.

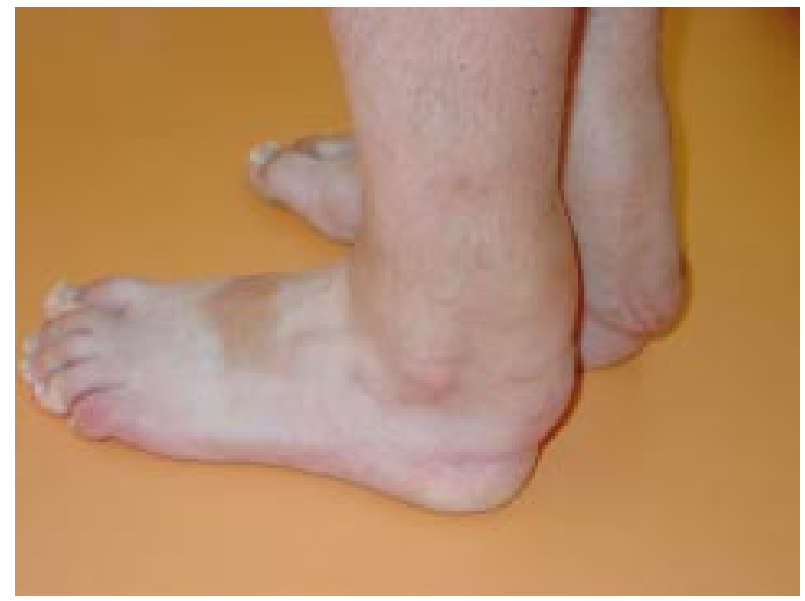

Figure 1 Enlargement of Achilles tendons.

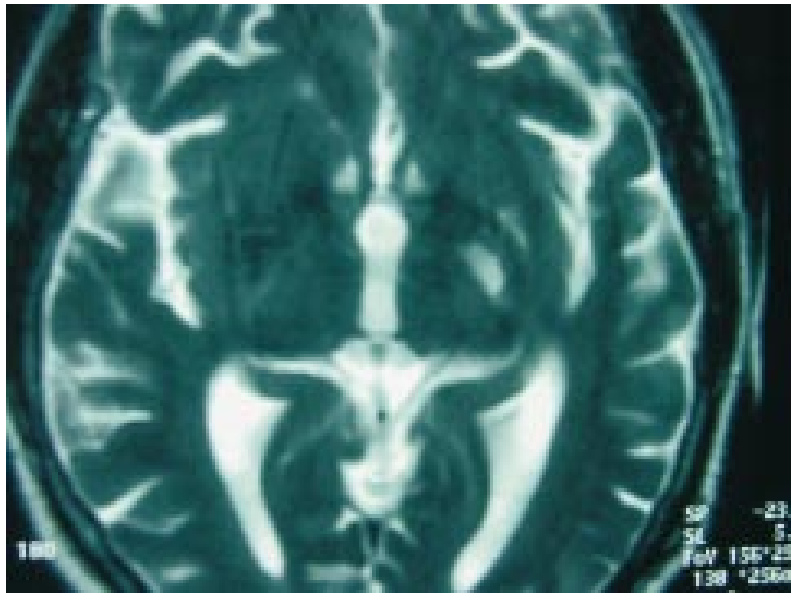

Figure 2 Axial T 2 weighted MR image showing left putaminal and capsular hyperintense lesion.

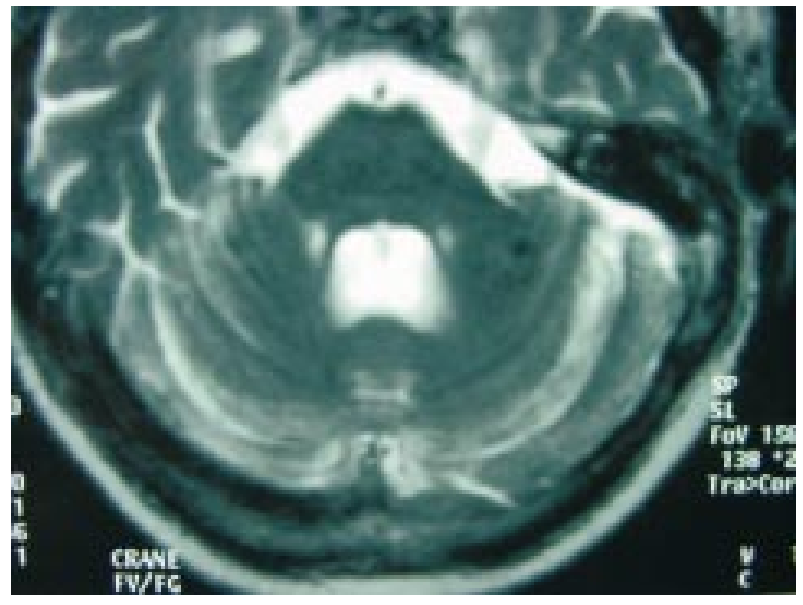

Figure 3 Axial T 2 weighted MR image showing focal bilateral symmetrical hyperintensive lesions in cerebellum. 